\title{
Psychosocial Constructs and Self-Reported Driving Restriction in the Candrive II Older Adult Baseline Cohort
}

Alexandra Jouk ${ }^{a}$, Holly Tuokko a , Anita Myers ${ }^{\mathrm{b}}$, Shawn Marshall ${ }^{\mathrm{c}}$, Malcolm Man-Son-Hing ${ }^{\mathrm{c}}$, Michelle M. Porter ${ }^{\mathrm{d}}$, Michel Bédard ${ }^{\mathrm{e}}$, Isabelle Gélinas ${ }^{\mathrm{fg}}$, Barbara Mazer ${ }^{\mathrm{fg}}$, Gary Naglie ${ }^{\text {hij }}$, Mark Rapoport $^{\mathrm{k}}$, \& Brenda Vrkljan ${ }^{1}$

on behalf of the Candrive II Research Team

${ }^{a}$ Centre on Aging, University of Victoria, PO Box 1700 STN CSC, Victoria BC, V8W 2Y2

${ }^{\mathrm{b}}$ School of Public Health \& Health Systems, University of Waterloo, Waterloo, Ont., Canada

${ }^{c}$ Ottawa Hospital Research Institute, University of Ottawa, Ottawa, Ont., Canada

${ }^{d}$ Faculty of Kinesiology and Recreation Management, University of Manitoba, Winnipeg, Man., Canada

${ }^{\mathrm{e}}$ Centre for Research on Safe Driving, Lakehead University, Thunder Bay, Ont., Canada

${ }^{\mathrm{f}}$ School of Physical \& Occupational Therapy, McGill University, Montreal, Que., Canada

${ }^{g}$ Centre de Recherche Interdisciplinaire en Réadaptation du Montréal Métropolitain, Montreal, Que., Canada

${ }^{\text {h }}$ Research Department, Toronto Rehabilitation Institute, University Health Network, Toronto, Ont., Canada

${ }^{\mathrm{i}}$ Department of Medicine and Rotman Research Institute, Baycrest Geriatric Health Care Centre, Toronto, Ont., Canada

${ }^{\mathrm{j}}$ Department of Medicine and Institute of Health Policy, Management and Evaluation, University of Toronto, Toronto, Ont., Canada

${ }^{\mathrm{k}}$ Department of Psychiatry, University of Toronto, Toronto, Ont., Canada

${ }^{1}$ School of Rehabilitation Science, McMaster University, Hamilton, Ont., Canada

Corresponding Author: Ms. Alexandra Jouk, Centre on Aging, University of Victoria PO BOX 1700, STN CSC, Victoria, BC, Canada V8W 2Y2, Tel (250) 532-6361, Email: ajouk@uvic.ca, fax: 250-721-6499 


\section{ABSTRACT}

Baseline data from a large cohort of 928 older drivers (aged 70 and older) in the Canadian Driving Research Initiative for Vehicular Safety in the Elderly (Candrive II) study permitted the examination of driving specific constructs of perceptions and attitudes based on Social Cognition Theory, the Transtheoretical Model of Behavior Change, and the Theory of Planned Behavior (e.g., driving confidence/comfort, perceived driving ability, and decisional balance) in relation to each other and to various self-reported measures of driving restrictions (i.e., kilometers driven, situations frequently encountered and avoided, degree of driving restriction). Although several of the psychosocial measures were correlated, the magnitudes of the correlations were low enough to suggest that multiple factors were being assessed. In addition, perceptions and attitudes were associated with driving exposure and patterns such that the more positive/comfortable older adults felt about driving and their driving ability, the greater their self-reported driving exposure (e.g., more kilometers driven per week, increased exposure to challenging driving situations/decreased avoidance of challenging situations, fewer restrictions). Conversely, older adults who expressed more negative views about driving, including feeling less comfortable behind the wheel, self-reported driving fewer kilometers per week, avoiding challenging driving situations, and engaging more in active driving restriction compared to more comfortable/confident older drivers. These findings support previous research and extend our understanding of the specific perceptions and attitudes that can contribute to behavior change, particularly for constructs derived from Social Cognitive Theory and the Transtheoretical Model.

Keywords: Older drivers, behavior change, psychosocial factors, perceptions, attitudes and beliefs, self-regulation 


\section{INTRODUCTION}

In our aging society, many older adults rely on personal vehicles to maintain their mobility and independence. Driving, in fact, continues to be the most popular means of transportation used by older adults in North America (Collia, Sharp, \& Giesbrecht, 2003; Kostyniuk \& Shope, 2003; Turcotte, 2012). This number is likely to increase in the next decade as the baby-boomer generation reaches older adulthood. As a result, older driver safety has never been a more important topic for researchers to address.

Concerns about older driver safety have been outlined in the extant literature due to aging and age-related disease that may put them at increased risk of crashes and other unsafe driving behaviors, such as changes in cognition, sensory function, and physical abilities (Anstey, Wood, Lord, \& Walker, 2005; Dobbs, 2002; Rizzo, McGehee, Dawson, \& Anderson, 2001). However, considerable evidence has demonstrated that some older adults voluntarily restrict their driving, thereby limiting exposure to risky driving situations, or stop driving altogether (Betz \& Lowenstein, 2010; Donorfio, D'Ambrosio, Coughlin, \& Mohyde, 2008; Hakamies-Blomqvist \& Wahlstrom, 1998). For instance, Betz and Lowenstein (2010) surveyed 9,684 drivers age 65 and older and found that $81.9 \%$ reportedly limited their driving, usually in bad weather, at night, on long trips, in traffic, or at high speeds. Understanding more about the factors underlying older driver behavior change, particularly self-regulatory practices (e.g., restriction, cessation), may be useful for developing intervention strategies that promote safety in this cohort.

A number of social science theories, including Bandura's Social Cognitive Theory (1986), the Transtheoretical Model of Behavior Change (Prochaska \& Velicer, 1997), and the Theory of Planned Behavior (Ajzen \& Fishbein, 1980; Ajzen, 1985), have been used to address behavior change in various contexts (e.g., smoking cessation, weight loss; Boyd \& Wandersman, 
1991; Budd, 1986; Marcus, Rossi, Selby, Niaura, \& Abrams, 1992; Prochaska, DiClemente, \& Norcross, 1992) and have more recently been applied to driving (Kowalski, Jeznach, \& Tuokko, 2014; Parker, 2002; Myers, Paradis \& Blanchard, 2008; Tuokko, McGee, \& Rhodes, 2006). These theories suggest that psychological processes like internal motivating factors (e.g., perceptions, attitudes, beliefs, self-efficacy, awareness, and intention) are necessary for behavior change and, as a result, are particularly relevant to understanding self-regulatory practices in older adults. According to the Transtheoretical Model of Behavior Change, beliefs (pros, cons, self-efficacy) and strategies to change act as mediators between external influences (e.g., information) and behavior to gradually move people through six distinct stages of readiness (precontemplative, contemplative, preparation, action, maintenance, termination). Similarly, the Theory of Planned Behavior posits that a person's intention to engage in a behavior is determined by instrumental and affective attitude toward the behavior (favorable or unfavorable instrumental/functional and emotional evaluations of driving), subjective norm (beliefs about how people he/she cares about will view the behavior in question), and perceived control (the person's perception of his/her ability to perform the behavior), and these beliefs act as a mediator in the behavior change process.

At least two models have been developed that explicitly outline these psychological processes as contributing to driver self-regulation (Lindstrom-Forneri, Tuokko, Garrett, \& Molnar, 2010; Rudman, Friedland, Chipman, \& Sciortino, 2006). In 2006, Rudman and her colleagues conducted focus groups with pre-senior drivers (age 55-64), senior drivers (age 6692), and senior ex-drivers (age 65-94) to better understand their perspectives concerning selfregulation. Themes that emerged were used to develop a preliminary model that highlights intrapersonal/psychological influences (e.g., awareness, comfort) on older driver self-regulation, 
in addition to interpersonal and environmental influences. Lindstrom-Forneri et al. (2010) expanded on the influence of psychological processes in driver self-regulating behaviors by specifically identifying attitudes and beliefs as a necessary mediating factor in this process. In their Driving as an Everyday Competence (DEC) model, a person's level of driving competence results from the interaction between individual and environmental factors at the global (i.e., social policies, location, presence of a disease or mental illness) and specific (i.e., physical health, cognitive status, emotional well-being, sensory state, and driving experience) contextual level and is mediated by attitudes, beliefs, self-efficacy, and awareness.

Given the importance of attitudes, beliefs, self-efficacy, and awareness in the selfregulatory process, several research groups have developed measures based on the principles of Social Cognitive Theory, the Transtheoretical Model of Behavior Change, and the Theory of Planned Behavior to more closely examine these constructs in older drivers (e.g., MacDonald, Myers, \& Blanchard, 2008; Baldock, Mathias, McLean, \& Berndt, 2006; Charlton, Oxley, Fildes, Oxley, Newstead, Koppel, \& O’Hare, 2006; Lindstrom-Forneri, Tuokko, \& Rhodes, 2007; Marottoli \& Richardson, 1998; Molnar \& Eby, 2008; Myers et al., 2008; Parker, MacDonald, Sutcliffe \& Rabbitt, 2001; Tuokko et al., 2006). Based on the construct of selfefficacy as defined by Social Cognitive Theory (Bandura, 1986), the Day and Night Driving Comfort Scales (DCS-D and DCS-N) were systematically developed with older drivers and subjected to rigorous psychometric examination using Rasch analysis to provide the most conceptually relevant, reliable, and valid measure of self-reported driving comfort/confidence available today (Myers et al., 2008; Blanchard \& Myers, 2010). Also tied to self-efficacy and awareness, perceived driving ability has been examined as a mediating factor in older driver selfregulation. The Perceived Driving Abilities (PDA) self-report scale, like the DCSs, was 
systematically developed with older drivers and found to have good psychometric properties (MacDonald et al., 2008; Blanchard \& Myers, 2010).

In addition to self-efficacy, decisional balance, which is predicated on weighing the pros and cons of engaging in various behaviors, has also been examined in the context of older driver self-regulation (Tuokko et al., 2006). The Transtheoretical Model was used as the basis for developing and pilot testing a self-report decisional balance instrument to examine the cognitive and motivational components of older drivers' readiness to change driving behavior. Four subscales were identified: (1) Pro-self (positive atttiudes concerning one's driving); (2) Pro-other (positive attitudes concerning one's driving in relation to others); (3) Con-self (negative attitudes concerning one's driving); and (4) Con-other (negative attitudes concerning one's driving in relation to others). Based on a pilot study with 40 drivers (aged 57 to 90,63\% women), individuals who possessed more positive attitudes about how their driving impacts others were less likely to be restricting their driving, whereas those who held more negative attitudes about their driving were more likely to be actively restricting their driving (Tuokko et al., 2006). These Decisional Balance subscales were found to have adequate internal consistency reliabilities.

The Decisional Balance scale was further modified by Lindstrom-Forneri and her colleagues (2007) to better align with the components outlined by the Theory of Planned Behavior. An additional five subscales were derived, including Affective Attitude (favorable or unfavorable emotional evaluations of driving), Instrumental Attitude (favorable or unfavorable instrumental/functional evaluation of driving), Perceived Control (perceived ability to drive), Subjective Norm (perceived social pressures to drive or not), and Intention (intent to continue driving). Structural equation modeling showed that Instrumental Attitude and Subjective Norm 
explained $30 \%$ of the variance associated with the intention to continue driving (LindstromForneri et al., 2007).

To date, the Driving Comfort Scales, the Perceived Driving Ability scale, and components of the Decisional Balance scale have only been examined with small samples and all three measures have not been used in the same study of older drivers (Blanchard \& Myers, 2010; Lindstrom-Forneri et al., 2007; MacDonald, Myers, \& Blanchard, 2008; Myers, Paradis, \& Blanchard, 2008; Tuokko et al., 2006). The Canadian Driving Research Initiative for Vehicular Safety in the Elderly (Candrive II) study provided the opportunity to examine these psychosocial measures simultaneously in a large sample (over 900 older drivers) at baseline to establish which, if not all, theories best captured the perceptions and attitudes of older drivers. Objectives of this investigation were threefold: (1) to examine the inter-relations between these measures, (2) to provide a description of how the various constructs (perceptions and attitudes,) contained in these measures relate to self-reported driving practices (i.e., kilometers driven, situations frequently encountered and avoided, degree of driving restriction), and (3) to refine our understanding of these measures to guide future studies when longitudinal data becomes available.

\section{MATERIALS AND METHODS}

\subsection{Participants}

All participants from the Candrive II baseline cohort were included in this examination. Full details on the study protocol and procedures are available in Marshall et al. (2013) and briefly described here. The prospective Candrive II study, which began in 2009, enrolled 928 older drivers from seven Canadian cities in four provinces (Ontario, Quebec, Manitoba, and British Columbia) for four years. To be included in the study, participants were required to be 
age 70 or older, drive a minimum of 4 times a week for at least a year with a valid license, and not have any medical contraindications to driving according to guidelines stipulated by the Canadian Medical Association (2006).

\section{2 $\quad$ Procedure}

Participants who met the eligibility criteria were scheduled for a baseline assessment. In addition to written consent for study participation, consent forms solicited permission to install an electronic recording device in their vehicle and access their driving records from licensing authorities. The baseline assessment included measures of health status, functioning, and driving habits and intentions. Participants were given a take-home package containing the psychosocial scales (described below) to be completed and returned by mail within two weeks.

\subsection{Measures}

\subsubsection{Predictor Variables}

Three sets of psychosocial measures examining various aspects of drivers' perceived comfort and ability, as well as their attitudes were treated as independent variables: (1) the Day and Night Driving Comfort Scales (DCSs; Myers et al., 2008), (2) the Perceived Driving Abilities (PDA) scale (MacDonald, Myers, \& Blanchard, 2008), (3) and the Decisional Balance Plus (DBP) scale (Lindstrom-Forneri et al., 2007; Tuokko et al., 2006).

The 13-item Day and 16-item Night DCS (Myers et al., 2008) are context specific (in relation to traffic flow, speed, road type) and have respondents rate their level of driving comfort on a 5-point percentage scale $(0 \%, 25 \%, 50 \%, 75 \%$, and $100 \%)$, from not at all comfortable to completely comfortable. Psychometric analysis of these measures showed them to be hierarchical, unidimensional, with good person and item reliabilities, as well as having temporal 
stability (Intraclass Correlation Coefficient (ICC) for DCS-Day $=.70$; ICC for DCS-Night $=.88$ ) and test-retest reliability (ICC $=.89$ and .92 , respectively) (Blanchard \& Myers, 2010).

The 15-item PDA scale (MacDonald et al., 2008) asks respondents rate their current perceived driving abilities (e.g., to see road signs at a distance, to see vehicles coming up alongside, to make an over the shoulder check) along a 4-point scale as "Poor", "Fair", "Good" or "Very Good." Possible total scores range from 0 to 45 with higher scores indicating more positive perceptions. Like the DCSs, the PDA scale has been shown to be hierarchical and unidimensional with good person $(r=.92)$ and item $(r=.82)$ reliabilities. The scale has good internal consistency $($ alpha $=.92)$ and moderate test-retest reliability $(\mathrm{ICC}=.65)($ Blanchard $\&$ Myers, 2010).

The DBP scale (Lindstrom-Forneri et al., 2007; Tuokko et al., 2006) asks older drivers rate their responses to various attitudinal statements about the self and others on a 5-point scale from "Strongly Agree" to "Strongly Disagree." This measure examines positive aspects of driving relevant for the individual (Pro-self) using 9 items (e.g., "Driving a vehicle is pleasurable"), with higher scores indicate fewer positive attitudes of the respondent's own driving). It also measures positive aspects of driving relevant for others (Pro-other) using 7 items (e.g., "Others count on me being able to drive"), with higher scores indicate fewer positive attitudes of the respondent's driving in relation to others). Additonally, the DBP scale examines negative aspects of driving relevant for the individual (Con-self) using 9 items (e.g., "The financial cost of maintaining a vehicle is an increasing concern of mine"), with higher scores indicate fewer negative attitudes of the respondent's own driving. Lastly, it examines negative aspects of driving relevant to others (Con-other) using 6 items (e.g., "My driving bothers other 
people"), with higher scores indicate fewer negative attitudes of the respondent's driving in relation to others).

Additional items on the DBP scale also were classified to reflect the Theory of Planned Behavior. The five subscales included: (1) Affective Attitude (examines affective evaluations of driving; e.g., "I am becoming more concerned about the unsafe and aggressive behavior of other drivers"; 3 items; higher scores indicate more favorable emotional evaluations of the respondent's own driving), (2) Instrumental Attitude (instrumental evaluations of driving; e.g., "Driving is important"; 3 items; higher scores indicate less favorable functional/instrumental evaluations of the respondent's own driving), (3) Perceived Control (perceived ability to drive; e.g., "Parking is becoming more difficult for me"; 3 items; higher scores indicate the greater perceived ability by the respondent to perform driving behaviors), (4) Subjective Norm (social pressure to drive; e.g., "My friends drive their vehicles regularly"; 3 items; higher scores indicate greater social pressure to drive), and (3) Intention (intention towards driving; e.g., "I plan to continue driving in the foreseeable future"; 2 items; higher scores indicate decreased intention to continue driving).

\subsubsection{Outcome Variables}

Four dependent variables were included in the analyses: (1) scores on the Situational Driving Frequency (SDF) scale, (2) scores on the Situational Driving Avoidance (SDA) scale, (3) self-reported kilometers driven, and (4) stage of behavior change. The SDF and SDA scales were developed with older drivers to assess self-reported practices (frequency and avoidance, respectively) concerning driving in challenging situations (MacDonald et al., 2008). On the SDF scale, respondents rate how frequently they engage in challenging driving situations on a 5-point scale from "Never" to "Very Often." Possible scores can range from 0 to 56, with higher scores 
indicating greater frequency of driving in challenging situations. On the SDA Scale, persons are given 1 point for each item (situation) checked. Possible SDA scores can range from 0 to 20, with higher scores indicating greater avoidance of challenging situations. Both the SDF and SDA have been shown to have good test-retest reliabilities over 7-14 days $(\mathrm{ICC}=.89$ and .86 , respectively) and high internal consistency with a Cronbach's alpha of .92 and .87 , respectively (Blanchard \& Myers, 2010).

To estimate driving exposure, participants were asked how many kilometers $(\mathrm{km})$, they drove on average over the past year by choosing one of the following: (1) less than $3,000 \mathrm{~km}$; (2) $3,001-5,000 \mathrm{~km}$; (3) $5,001-10,000 \mathrm{~km}$; (4) $10,001-15,000 \mathrm{~km}$, or (5) greater than $15,001 \mathrm{~km}$ a year. Classification for the kilometer range was derived from work by Langford as well as Staplin (Langford, Koppel, McCarthy, \& Srinivasan, 2008; Staplin, Gish, \& Joyce, 2008).

The final outcome variable included in this study was participants' current stage of behavior change, as conceptualized by the Transtheoretical Model of Behavior Change (Prochaska \& Velicer, 1997). Since eligibility required participants to be active drivers, not all stages of the Transtheoretical Model could be examined (e.g., termination/cessation) using baseline data. Individuals were asked whether or not they restrict their driving under a number of different conditions (e.g., limit driving to the day, avoid driving in inclement weather). Based on the Transtheoretical Model's definition for the stages, participants who did not report restricting their driving under any condition were considered to reflect the "pre-action" (i.e., "precontemplative", "contemplative", "preparation") stages of the Transtheoretical Model, while participants who reported actively restricting their driving under any condition were considered to fall under the "action" stage.

\subsection{Statistical Analysis}


Pearson's Correlation analyses were conducted to examine the associations between scores on the various measures. Pearson correlations were also used to examine the relations between the psychosocial scores (Day and Night DCSs, DBP) and how frequently older adults drove in and avoided challenging driving situations as measured by the SDF and SDA scale, respectively. The relations between scores on the psychosocial measures and mileage driven and level of driving restriction/stage of change (not-restricting or restricting) were also examined using one-way analyses of variance (ANOVA) with Tukey's HSD post-hoc comparisons. Although scores on many of the psychosocial measures were skewed, Pearson's correlations and ANOVAs were used rather than Spearman's Rho and Kruskal-Wallis tests as the results did not differ between the two methods and for ease of reporting. All analyses were conducted using SPSS Version 21 (IBM Corp, 2012). Given the large number of comparisons conducted, an alpha level of $\mathrm{p}<.01$ was considered indicative of significant effects.

\section{RESULTS}

\subsection{Sample Characteristics}

A total of 928 participants completed the series of background and driving habits questionnaires, as well as most of the psychosocial measures described above. The total sample ranged in age from 70 to 94 years $(M=76.2, S D=4.9)$ and included 351 females $(37.8 \%)$ and 577 males (62.2\%). Not all subjects fully completed the psychosocial or driving restriction measures; variations in sample size are noted in each section of the results.

\subsection{Associations between Scores on the Psychosocial Measures}

Table 1 presents the inter-correlations between scores on the psychosocial measures, as well as associations with two of the driving indicators (SDF and SDA scores). Scores on the driving comfort (the DCS-Day and DCS-Night) scales were highly correlated and both were 
moderately associated with the scores on the perceived driving abilities (PDA) scale. Scores on all three perception measures were moderately related to the two indicators of driving restrictions (SDF and SDA scores).

Similarly, scores on the various subscales of the DBP were moderately correlated to one another. Scores on the DBP were significantly related to scores on the perception measures (DCS and PDA scales), as well as to scores on the measures of restrictions (SDA and SDF) in the expected directions, with the following exceptions: DCS-Day/Instrumental Attitude and DCSNight/Instrumental Attitude.

\section{-Insert Table 1 Here-}

\subsection{Relations between Driving Frequency and Avoidance and Psychosocial Measures}

Pearson's correlation analyses were used to examine the relations between each psychosocial measure and how frequently older adults engaged in challenging driving situations as measured by the SDF scale. Results are displayed in Table 1. There were significant positive correlations between the DCS-Day, DCS-Night, PDA, DBP Con-self, DBP Con-other, DBP Affective Attitude, DBP Perceived Control, and DBP Subjective Norm measures and the SDF scale. Higher driving comfort levels during both the day and night were associated with increased frequency of challenging driving situations. Individuals with higher perceived driving abilities (PDA and DBP Perceived Control) also engaged in more challenging driving situations. Drivers who held more negative views of driving relevant to themselves (DBP Con-self and Affective Attitude) and who believed others viewed their driving negatively (DBP Con-other), as indicated by lower scores on these three subscales, engaged in fewer challenging driving situations. Lastly, individuals who felt greater pressure from others to continue driving (higher scores on the DBP Subjective Norm subscale), engaged in more challenging driving situations. 
Significant negative correlations were found between the DBP Pro-self, Pro-other, Instrumental Attitude, and Intention subscales and the SDF. Individuals who positively perceived driving with respect to themselves and others (lower scores on the DBP Pro-self and Pro-other subscales) engaged in a greater number of challenging driving situations. More specifically, individuals who positively perceived driving with respect to the functional aspects related to driving (lower scores on the DBP Instrumental Attitude subscale) also engaged in a greater number of challenging driving situations. Additionally, individuals who planned to continue driving in the near future (lower scores on the DBP Intention subscale) engaged in a greater number of challenging driving situations.

Pearson's correlation analyses were also conducted to examine the relations between each psychosocial measure and how often certain challenging driving situations were avoided as indicated by scores in the SDA scale (Table 1). A significant positive correlation was found between the DBP Intention subscale and the SDA scale, indicating that individuals with more of an intention to continue driving in the near future avoided fewer challenging driving situations than individuals with less of an intention to continue driving. Significant negative correlations were found between the DCS-Day, DCS-Night, PDA, and DBP Con-self, Con-other, Affective Attitude, and Perceived Control scales and the SDA scale. Greater comfort with driving during the day and night was associated with less avoidance of challenging driving situations. The better individuals perceived their driving abilities to be (PDA and DBP Perceived Control), the less they avoided challenging driving situations. Those who held more negative views of their own driving (DBP Con-self and Affective Attitude) and who believed others viewed their driving negatively (DBP Con-other), as indicated by lower scores on these three subscales, avoided more 
challenging driving situations. No significant associations were found between the DBP Pro-self, Pro-other, Instrumental Attitude subscales, and the SDA scale.

\subsection{Associations between Psychosocial Scores and Kilometers Driven}

One-way ANOVAs, with Tukey's HSD post-hoc comparisons when the overall F was significant, were used to examine the relations between scores on each psychosocial measure and mileage driven over the past year. The constructs showing a significant association with mileage are shown in Table 2. Driving comfort levels during the day and night, as measured by the DCSDay and DCS-Night scales, respectively, significantly differed in relation to the number of kilometers driven such that drivers with greater comfort levels on the road reported more kilometers driven.

Older adults' perceived driving abilities, as measured by the PDA scale and DBP Perceived Control subscale, significantly differed between the range of kilometers driven per year, such that those driving the most kilometers had higher perceived driving abilities compared to those driving moderate distances.

Individuals who drove the most kilometers per year felt more positively about their driving, as indicated by a lower score on the DBP Pro-self subscale, than individuals who drove fewer kilometers. Similarly, individuals with low yearly mileage rates felt there were more negative aspects associated with their driving (Con-self) than individuals who drove more kilometers in a year. Overall, older adults who drove fewer kilometers per year had significantly less favorable views of driving than individuals who drove the most, as measured by the DBP Affective Attitude subscale. Finally, individuals who drove fewer kilometers per year perceived less pressure to continue driving (as measured by the DBP Subjective Norm subscale) than older adults who drove the most per year. 
- $\quad$ Insert Table 2 Here -

\subsection{Relations between Stages of Change and Psychosocial Measures}

Relations between each psychosocial measure and whether or not participants were restricting their driving were examined using one-way ANOVAs (Table 3). Apart from the DBP Instrumental Attitude subscale, significant associations were found between each of the other measures.

Individuals who did not restrict their driving were substantially more comfortable driving during both the day and night compared to individuals who restricted their driving during the day and night as indicated by scores on the DCS-Day and DCS-Night. They also perceived their driving abilities more favorably than older drivers who restricted their driving (as indicated by scores on the PDA and DBP Perceived Control subscale), and had more positive attitudes towards driving (scores on the DBP Pro-self subscale) compared to individuals who restricted their driving. Additionally, those who did not restrict their driving held more positive views that their driving was relevant to others (DBP Pro-other) compared to restricting drivers. Nonrestricting drivers felt there were less negative aspects associated with their driving (DBP Conself) compared to restricting drivers. Older adults who restricted their driving believed others viewed their driving more negatively than non-restricting drivers, as indicated by scores on the DBP Con-other subscale. Similarly, responses from the DBP Subjective Norm subscale indicated that restricting drivers perceived less pressure from others to drive than non-restricting drivers. Scores on the DBP Affective Attitude subscale indicated that, overall, individuals who did not restrict their driving held more favorable affective views of driving than restricting drivers. Finally, scores on the DBP Intention subscale indicated that older adults not restricting their 
driving had greater intention to continue driving in the foreseeable future than individuals who were already actively restricting their driving.

- Insert Table 3 Here -

\section{DISCUSSION}

The Candrive II baseline data used in this study permitted the examination of multiple psychosocial measures and indicators of restricted driving practices in a large sample. Overall, we found that most of the psychosocial measures were significantly related to one another. According to Cohen (1992), correlations of .10, .30, and .50 represent small, medium, and large effect sizes, respectively. In these data, the majority of the inter-correlations between the psychosocial measures represented small to medium sizes. This could be expected given the consistency in format (e.g., self-report) and general content (e.g., perceptions, attitudes and beliefs about driving) of the psychosocial measures. In addition, social desirability bias could have shaped participants' responses and contributed to inflated correlations. It is important to note, however, that fairly weak correlations were found between the different sets of measures, which may reflect underlying differences in the theoretical constructs of each measure.

However, several strong effect sizes were noted. The strongest effect size was found for the correlation between the Day and Night Driving Comfort Scales, which is consistent with previous literature (MacDonald et al., 2008; Myers et al., 2008; Blanchard \& Myers, 2010). It is expected that these two scales are related, however research has repeatedly shown that night scores are lower than day scores, which was also supported in this study (Blanchard \& Myers, 2010; Myers et al., 2008). Other strong magnitudes of association were found between the DBP Pro-self and Instrumental Attitudes subscales, the DBP Perceived Control and Con-self subscales, the DBP Con-other and Subjective Norm subscales, and the DBP Con-self and 
Affective Attitude subscales. In each pair of correlated subscales, several of the same items were used to create the scale. The high effect sizes likely reflect the similarity in item content.

More moderate, but still strong, effect sizes were found between the DCS-Day/PDA, DCS-Day/SDA, DCS-Night/PDA, DCS-Night/SDA, DCS-Night/SDF, SDA/SDF, DBP Proself/DBP Pro-other, DBP Con-self/DBP Con-other, DBP Pro-other/DBP Instrumental Attitude, and DBP Con-other/DBP Perceived Control. For the scales based on the Social Cognitive Theory, the magnitude of the correlations found in this study were similar to the results reported in several other studies (Blanchard \& Myers, 2010; MacDonald et al., 2008; Myers, Trang, \& Crizzle, 2011). For the scales based on the Transtheoretical Model, the magnitude of the Proself/Pro-other correlation was comparable to the correlation reported by Tuokko and her colleagues (2006). The magnitude of the Con-self/Con-other correlation reported by Tuokko and her colleagues, however, was much higher compared to this study ( $r=.772$ vs. $r=.596)$. The differences in the magnitude between this study and the study by Tuokko and her colleagues may be due to the more focused age range used in the Candrive II study.

When examining the magnitudes of all the inter-correlations between the psychosocial scales used in this study, the scales based on the Social Cognitive Theory and Transtheoretical Model tend to have higher correlations than the scales based on the Theory of Planned Behavior. The scales based on the Social Cognitive Theory and Transtheoretical Model contain more items than the scales based on the Theory of Planned Behavior, which may account for this difference. It may be, however, that the constructs captured by the scales based on the Social Cognitive Theory (e.g., self-efficacy) and the Transtheoretical Model (e.g., pros and cons related to the behavior) may be more relevant to understanding driving than the constructs of the Theory of Planned Behavior, which emphasizes intention to perform the behavior within the context of the 
amount of control the individual possesses over the behavior. Given that driving behavior changes gradually with increasing restrictions as to where and when driving takes place, and likely occurs in stages (Kowalski et al., 2014), the Transtheoretical Model may more closely mirror this process than the Theory of Planned Behavior.

Additional results of this study indicated that perceptions and attitudes,were associated with self-reported restrictions in driving practices such that the more positive/comfortable older adults felt about driving the more they reported driving overall $(\mathrm{km}$ per year) and in more challenging situations. Conversely, older adults who reported more negative views about driving, including feeling less comfortable behind the wheel, self-reported driving fewer kilometers per year, avoided more challenging driving situations, and actively restricted their driving compared to more comfortable/confident older adults. Previous research also found that perceptions (lower driving comfort and poorer perceived abilities) are associated with increased self-regulatory practices (Blanchard \& Myers, 2010; Gwyther \& Holland, 2012; Myers et al., 2011). In 2010, Blanchard and Myers monitored actual driving patterns in 61 older drivers using in-vehicle devices in addition to examining perceptions of driving using the DCSs and the PDA scale. They found that older adults who reported less comfort driving and poorer perceived driving abilities reduced their exposure (kilometers and duration) during the day and night and in challenging driving situations. Gwyther and Holland (2012) observed that negative attitudes toward driving and anxiety when driving independently predicted self-reported avoidance of difficult driving situations; however, this study only used one item to assess attitudes toward driving, whereas the measures used in this study used multi-item scales.

Given the consistency with previous research, this study supports the use of the DCSs, PDA, SDF, SDA, and DBP measures to examine attitudes and beliefs among older drivers. 
Although items are similar across the measures, which can contribute to participant burden, the scales measure different constructs and different driving contexts. For example, the DBP scales examine outcomes and incorporate interpersonal factors, while the DCSs and PDA scale look more at process and intrapersonal factors. In addition, different underlying theories are represented in the various measures. Depending on the theoretical position and the specific context a study requires, a researcher may choose to use just some or all of the measures. However, specific focus should be given to the scales derived from the Social Cognitive and Transtheoretical Model as these scales had higher inter-correlations than the scales based on the Theory of Planned Behavior. In addition, several of the scales derived from the Theory of Planned Behavior are quite similar, but much more broad, compared to the other scales (e.g., the Affective Attitude scale encompasses the Pro-self and Con-self scales and the Subjective Norm scale encompasses the Pro-other and Con-other scales).

This detailed analysis of the relations among attitudes, perceptions, and driving restrictions increases our understanding of the psychological processes underlying driver selfregulation among older adults. In the Driving as an Everyday Competence model (LindstromForneri et al., 2010), psychosocial factors, such as perceptions and attitudes about driving, act as mediators that influence driving behaviors. The current study provides evidence in support of this model and extends our understanding of the specific attitudes and beliefs that can contribute to behavior change.

The Candrive II baseline sample was largely comprised of healthy adults aged 70 years and older who were active drivers. As a result, ceiling effects may have limited the variability in some of the measures used in this study. In addition, the distributions of scores on most of the measures examined were skewed, thus the findings must be viewed with caution. 
Caution must also be exercised when interpreting the results, as this study obtained older drivers' self-report of their own abilities, which may not be an accurate reflection of actual driving performance (Horswill, Anstey, Hatherly, Wood, \& Pachana, 2011). As longitudinal data becomes available in the coming years, we will be able to examine how accurate Candrive II participants' self-reported driving behaviors correlate with actual driving patterns since invehicle recording devices monitored and tracked participants' driving.

Additionally, it is anticipated that as the years progress, some Candrive II participants will change their driving practices or stop driving, allowing us to extend our examination of those who restrict or not and look more closely at the stages of change as conceptualized by the Transtheoretical Model. More specifically, we hope to understand the factors influencing drivers' decisions to move through the various stages, from pre-contemplation to maintenance. Longitudinal data will also allow us to examine which psychosocial factors contribute to driver self-regulatory behavior as well as how attitudes and perceptions may change with the development of health-related conditions and involvement in crashes. 


\section{ACKNOWLEDGEMENTS}

We thank the older driver participants and the Research Associates at each data collection site without whose valuable contributions this research would not be possible. We acknowledge with thanks Candrive's key partners: the National Association of Federal Retirees, Canadian Association for the Fifty-Plus (CARP), Municipal Retirees Organization Ontario, Canadian Council of Motor Transport Administrators and Transport Canada. This paper was prepared on behalf of the Candrive II Research Team: Shawn C. Marshall, Malcolm Man-Son-Hing, Paul

Boase, Michel Bédard, Anna Byszewski, Ann B. Cranney, Hillel M. Finestone, Sylvain Gagnon, Isabelle Gélinas, Michel J, Johnson, Nicol Korner-Bitensky, Linda C. Li, Barbara L. Mazer, Frank J. Molnar, Jeannette Montufar, Anita M. Myers, Gary Naglie, Jan A. Polgar, Michelle M. Porter, Mark J. Rapoport, Ian G. Stiell, Holly A. Tuokko, Brenda H. Vrkljan, George A. Wells. This work was funded by a Team Grant from the Canadian Institutes of Health Research awarded to Candrive entitled "The CIHR Team in Driving in Older Persons (Candrive II) Research Program" (grant 90429). Candrive's aim is to improve the health, safety, and quality of life of Canada's older drivers. Candrive's vision is to establish a national multi-disciplinary collaborative approach to identify, analyze, and examine issues pertaining to the safe operation of vehicles by older persons. Dr. Naglie is supported by the George, Margaret and Gary Hunt Family Chair in Geriatric Medicine, University of Toronto. 


\section{REFERENCES}

Ajzen, I. (1985). From intentions to actions: A theory of planned behavior. In J. Kuhl and J. Beckman (eds) Action Control: From Cognition to Behavior. Heidelberg: Springer.

Ajzen, I. \& Fishbein, M. (eds). (1980). Understanding Attitudes and Predicting Social Behavior. Englewood Cliffs, NJ: Prentice-Hall.

Anstey, K. J., Wood, J., Lord, S., \& Walker, J. G. (2005). Cognitive, sensory and physical factors enabling driving safety in older adults. Clinical Psychology Review, 25(1), 45-65.

Baldock, M.R.J., Mathias, J.L., McLean, A.J., \& Berndt, A. (2006). Self-regulation of driving and its relationship to driving ability among older adults. Accident Analysis and Prevention, 38, 1038-1045.

Bandura, A. (1986). Social foundations of thought and action: A social cognitive theory. Englewood Cliffs: Prentice-Hall.

Betz, M.E., \& Lowenstein, S.R. (2010). Driving patterns of older adults: Results from the second injury control and risk survey. Journal of the American Geriatric Society, 58, 1931-1935.

Blanchard, R.A., \& Myers, A.M. (2010). Examination of driving comfort and self-regulatory practices in older adults using in-vehicle devices to assess natural driving patterns. Accident Analysis and Prevention, 42, 1213-1219.

Boyd, B. \& Wandersman, A. (1991). Predicting undergraduate condom use with the Fishbein and Ajzen and the Triandis Attitude-Behavior models: Implications for public health interventions. Journal of Applied Social Psychology, 21(22): 1810-1830.

Budd, R.J. (1986). Predicting cigarette use: The need to incorporate measures of salience in the Theory of Reasoned Action. Journal of Applied Social Psychology, 16(8), 663-685.

Canadian Medical Association. (2006). Determining medical fitness to operate motor vehicles: CMA driver's guide. 7th ed. Ottawa: Available from http://www.cma.ca/multimedia/CMA/Content_Images/Inside_cma/WhatWePublish/Driv ers_Guide/Contents_e.pdf. Accessed March 27, 2012.

Charlton, J.L., Oxley, J., Fildes, B., Oxley, P., Newstead, S., Koppel, S., \& O’Hare, M. (2006). Characteristics of older drivers who adopt self-regulatory driving behaviours. Transportation Research Record, 9, 363-373.

Cohen, J. (1992). A power primer. Psychological Bulletin 112(1), 155-159.

Collia, D. V., Sharp, J., \& Giesbrecht, L. (2003). The 2001 national household travel survey: A look into the travel patterns of older Americans. Journal of Safety Research, 34, 461-470.

Dobbs, B. (2002). Medical conditions and driving: Current knowledge. (Report No. DTNH2294-G-05297) U.S. Department of Transportation, National Highway Traffic Safety Administration, Washington, DC. 
Donorfio, L.K.M., D’Ambrosio, L.A., Coughlin, J.F., \& Mohyde, M. (2008). Health, safety, selfregulation and the older driver: It's not just a matter of age. Journal of Safety Research, $39,555-561$.

Gwyther, H., \& Holland, C. (2012). The effect of age, gender and attitudes on self-regulation in driving. Accident Analysis And Prevention, 45, 19-28.

Hakamies-Blomqvist, L. \& Wahlstrom, B. (1998). Why do older drivers give up driving? Accident Analysis and Prevention, 30, 305-312.

Horswill, M.S., Anstey, K.J., Hatherly, C., Wood, J.M., \& Pachana, N.A. (2011). Older drivers' insight into their hazard perception ability. Accident Analysis and Prevention, 43, 21212127.

IBM Corp. (2012). IBM SPSS Statistics for Windows, Version 21.0. Armonk, NY: IBM Corp.

Kostyniuk, L. P., \& Shope, J. T. (2003). Driving and alternatives: older drivers in Michigan. Journal of Safety Research, 34(4), 407-414.

Kowalski, K., Jeznach, A., \& Tuokko, H.A. (2014). Stages of driving behavior change within the Transtheoretical Model. Journal of Safety Research, 50, 17-25.

Langford, J., Koppel, S., McCarthy, D., \& Srinivasan, S. (2008). In defence of the 'low mileage bias'. Accident Analysis and Prevention, 40(6), 1996-1999.

Lindstrom-Forneri, W., Tuokko, H. A., Garrett, D., \& Molnar, F. (2010). Driving as an everyday competence: A model of driving competence and behavior. Clinical Gerontologist: The Journal Of Aging And Mental Health, 33(4), 283-297.

Lindstrom-Forneri, W., Tuokko, H., \& Rhodes, R. (2007). "Getting Around Town": A preliminary investigation of the theory of planned behavior and intent to change driving behaviors among older adults. Applied Gerontology, 26(4), 385-398.

MacDonald, L., Myers, A.M., \& Blanchard, R.A. (2008). Correspondence among older drivers' perceptions, abilities and driving behaviors. Topics in Geriatric Rehab, 24, 239-252.

Marcus, B.H., Rossi, J.S., Selby, V.C., Niaura, R.S., \& Abrams, D.B. (1992). The stages and processes of exercise adoption and maintenance in a worksite sample. Health Psychology, 11(6), 386-395.

Marottoli, R. A., \& Richardson, E. D. (1998). Confidence in, and self-rating of, driving ability among older drivers. Accident Analysis And Prevention, 30(3), 331-336.

Marshall, S., Man-Son-Hing, M., Charlton, J., Koppel, S., Langford, J., Tuokko, H., et al. (2013). Protocol for Candrive II/OzCandrive, a multicentre prospective older driver cohort study. Accident Analysis and Prevention. Advance online publication.

Molnar, L.J., \& Eby, D.W. (2008). The relationship between self-regulation and driving related abilities in older drivers: An exploratory study. Traffic Injury Prevention, 9, 314-319. 
Myers, A.M., Paradis, J., \& Blanchard, R.A. (2008). Conceptualizing and measuring confidence in older drivers: Development of the Day and Night Driving Comfort Scales. Arch Phys Med Rehab, 89, 630-640.

Myers, A.M., Trang, A., \& Crizzle, A.M. (2011). Naturalistic study of winter driving practices by older men and women: Examination of weather, road conditions, trip purposes, and comfort. Canadian Journal on Aging, 30(4), 577-589.

Parker, D. (2002). Changing drivers' attitudes to speeding: Using the Theory of Planned Behavior. In D. Rutter, L. Quine (Eds.), Changing health behavior: Intervention and research with social cognition models (pp. 138-152). Maidenhead, BRK England: Open University Press.

Parker, D., Macdonald, L., Sutcliffe, P., \& Rabbitt, P. (2001). Confidence and the older driver. Ageing and Society, 21(02), 169-182.

Prochaska, J.O., DiClemente, C.C., \& Norcross, J.C. (1992). In search of how people change: Applications to addictive behaviors. American Psychologist, 47(9), 1102-1114.

Prochaska, J.O. \& Velicer, W.F. (1997). The transtheoretical model of health behavior change, American Journal of Health Promotion, 21(1), 38-48.

Rizzo, M., McGehee, D.V., Dawson, J.D., \& Anderson, S.N. (2001). Simulated car crashes at intersections in drivers with Alzheimer disease. Alzheimer Disease and Associated Disorders, 15(1), 10-20.

Rudman, D.L., Friedland, J. Chipman, M., \& Sciortino, P., (2006). Holding on and letting go: The perspectives of pre-seniors and seniors on driving self-regulation in later life. Canadian Journal on Aging, 25(1), 65-76.

Staplin, L., Gish, K., \& Joyce, J. (2008). 'Low mileage bias' and related policy implications - A cautionary note. Accident Analysis and Prevention, 40, 1249-1252.

Tuokko, H., McGee, P., Rhodes, R.E. (2006). Decisional balance and readiness to change driving behavior in older adults: A pilot study. Physical \& Occupational Therapy in Geriatrics, 24(3), 1-12.

Turcotte, M. (2012). Profile of seniors' transportation habits. Statistics Canada, 11(008), 1-16. 